\title{
UNA MIRADA A LA PERMANENCIA DE LA POBLACIÓN ESTUDIANTIL DE TRABAJO SOCIAL
}

A LOOK TO THE PERMANENCY OF THE SOCIAL WORK STUDENT POPULATION

\section{Volumen 13, Número 3}

Setiembre - Diciembre

pp. $1-25$

Este número se publicó el 30 de setiembre de 2013

Gabriela Regueyra Edelman

Carolina Rojas Madrigal

Revista indizada en REDALYC, $\underline{\text { SCIELO }}$

Revista distribuida en las bases de datos:

CATÁLOGO DE LATINDEX, IRESIE, CLASE, DIALNET, DOAJ, E-REVIST@S, SHERPA/ROMEO, QUALIS, MIAR

Revista registrada en los directorios:

ULRICH'S, REDIE, RINACE, OEI, MAESTROTECA, PREAL, CLASCO 


\title{
UNA MIRADA A LA PERMANENCIA DE LA POBLACIÓN ESTUDIANTIL DE TRABAJO SOCIAL
}

\author{
A LOOK TO THE PERMANENCY OF THE SOCIAL WORK STUDENT POPULATION
}

\author{
Gabriela Regueyra Edelman ${ }^{1}$ \\ Carolina Rojas Madrigal ${ }^{2}$
}

\begin{abstract}
Resumen: El artículo sintetiza una investigación realizada en la Escuela de Trabajo Social de la Universidad de Costa Rica sobre la permanencia de la población estudiantil en la carrera durante el periodo 2002-2008. La investigación fue descriptiva: se recogió información demográfica, académica y socioeconómica de la población y se realizó un seguimiento de cohortes de estudiantes, utilizando como base la información que la Universidad tiene almacenada en el Sistema de Aplicaciones Estudiantiles. El estudio permite mostrar la importancia de acercarse a la permanencia como proceso macro que vive cada estudiante desde su primer contacto con la Universidad hasta la obtención de un título. Se logran dilucidar aspectos por los cuales el estudiantado obtiene los títulos de bachillerato y licenciatura en el tiempo estipulado. Se concluye que tienen mayor probabilidad de lograrlo quienes ingresaron a la universidad y a la carrera en el mismo año, a una edad entre 17 y 18 años, quienes habitan en zonas cercanas a la Sede Rodrigo Facio, estudiaron en colegios académicos, contaron con beca por condición socioeconómica y beneficios complementarios, matricularon solamente cursos de Trabajo Social, aprobaron más de 15 créditos por ciclo lectivo regular y llevaron cursos en el tercer ciclo lectivo. Además, se logró revisar información de población que no continúa en la carrera, la cual corresponde a un $22 \%$ del grupo estudiado. La principal razón de no continuación se relaciona con haber ingresado a una carrera que no era de su interés.
\end{abstract}

Palabras clave: DESERCIÓN, PERMANENCIA, ESTUDIANTES DE TRABAJO SOCIAL, POBLACIÓN ESTUDIANTIL, GRADUACIÓN, UNIVERSIDAD DE COSTA RICA

\begin{abstract}
The article synthesizes an investigation realized at the Social Work School, University of Costa Rica about the permanence of its student population during the period 2002-2008. The research was descriptive: the information on the themes of demographic, academic and socioeconomic behavior of the population was captured and the student cohorts were traced, based on the information stored at the University Student Applications System. The study established the importance of approaching permanence as a macro process that the student population experience since their first contact with the University until they attain an academic degree. It clarifies aspects on how the undergraduate and graduate degrees in the stipulated time are achieved. It highlights that the students that are more likely to achieve this objectives, were those admitted to the University and the career at the same year, at an age between 17 and 18 years old, also, those who lived in areas near Rodrigo Facio Campus, studied in academic high-schools, received a scholarship and additional benefits per socioeconomic condition, enrolled only in Social Work courses, approved more than 15 credits per school regular period and matriculated courses on the third school period. It also captures information about the people that dropout the career, which corresponds to $22 \%$ of the population on study. The main reason for not continuing is related to entering a career that does not interest them.
\end{abstract}

Key words: DROPOUT, PERMANENCY, SOCIAL WORK STUDENTS, STUDENT POPULATION, GRADUATION, UNIVERSITY OF COSTA RICA

\footnotetext{
${ }^{1}$ Profesora Escuela de Trabajo Social de la Universidad de Costa Rica. Investigadora en el Instituto de Investigación en Educación, de la misma universidad. Magister en Educación, Licenciada en Trabajo Social, ambos títulos de la Universidad de Costa Rica.Dirección electrónica: gabriela.regueyra@ucr.ac.cr

2 Docente e Investigadora, Escuela de Trabajo Social y Departamento de Investigación y Evaluación Académica del Centro de Evaluación Académica, Universidad de Costa Rica. Licenciatura y Maestría en Trabajo Social de la Universidad de Costa Rica.Dirección electrónica: carolina.rojas@ucr.ac.cr
}

Artículo recibido: 19 de febrero, 2013

Aprobado: 19 de agosto, 2013 


\section{Antecedentes de la investigación}

El proyecto de investigación que sustenta el presente artículo surge de las reflexiones en la Escuela de Trabajo Social de la Universidad de Costa Rica, en procura del mejoramiento continuo.

Esta Unidad Académica, con siete décadas de dedicarse a la formación de profesionales, ha instaurado, desde las últimas cuatro, una cultura de autoevaluación, que ha permitido una revisión constante de los fundamentos de su propuesta curricular, así como del norte que se sigue.

Esta cultura proveyó la plataforma para iniciar el proceso de autoevaluación para la acreditación con el Sistema Nacional de Acreditación de la Educación Superior -SINAESen el año 1998. Para llegar a esta decisión, se reflexionó sobre las implicaciones de este proceso, el cual no se asumió de forma acrítica ni ingenua, ni para alinearse a las tendencias neoliberales del control de la calidad, sino con el interés de continuar con la revisión y autoevaluación (Molina y Ruiz, 2001).

La acreditación otorgada para el periodo 2001-2005 permitió implementar una serie de medidas de mejora. Posteriormente, se inició el proceso de autoevaluación para la reacreditación, también con el SINAES, vigente en el periodo 2006 - 2014.

En este último proceso de autoevaluación se ubicó como un tema neurálgico la situación del estudiantado, de forma que, en el Plan de mejoramiento para la reacreditación, se logró explicitar una serie de preocupaciones relacionadas con los tiempos de graduación y con el perfil y los comportamientos de deserción de las y los estudiantes. Se llegaron a plantear tres acciones:

1. Evaluación del proceso de elaboración de diseños, ejecución y de defensa oral de los Trabajos Finales de Graduación (TFG), para definir estrategias que permitan disminuir los tiempos de graduación sin descuidar la calidad de las investigaciones.

2. Monitoreo de los procesos de cumplimiento de los tiempos de graduación y de las disposiciones emanadas de la Sección de Investigación, la Comisión de Investigación y de Trabajos Finales de Graduación, así como del Comité Ético Científico de la Universidad de Costa Rica.

3. Desarrollo de un proyecto de investigación sobre el perfil socio-laboral y académico de los y las estudiantes y de los comportamientos de la deserción estudiantil. (Universidad de Costa Rica, Escuela de Trabajo Social, 2006, p. 30) 
Estas acciones se definen debido a que, en el proceso de autoevaluación mencionado, se evidenció que "hasta el momento se desconocen los factores asociados a la deserción estudiantil y a la duración de los Trabajos Finales de Graduación en la carrera" (Universidad de Costa Rica, Escuela de Trabajo Social, 2006, p. 30).

El primer acercamiento a estas acciones se da desde la Comisión de Evaluación y Orientación de la Unidad Académica, instancia en la que se diseñó y aplicó una encuesta sobre intención de traslado a otras carreras al 62\% del estudiantado matriculado en 2008. La tabulación de dicha encuesta se presentó en la sesión II-2009 de la Asamblea de Escuela, a partir de la cual dicho órgano conoce y analiza ampliamente los resultados, los valora como un primer acercamiento al tema y, en la misma discusión que se genera, se plantean interrogantes. Finalmente se acordó que estas fuesen tratadas en una investigación (Universidad de Costa Rica, Escuela de Trabajo Social, 2009).

Un asunto importante respecto de este primer acercamiento al tema es que, si bien parte de un esfuerzo realizado por una Comisión para elaborar, aplicar y tabular un instrumento, es evidente que captar su complejidad trasciende las posibilidades de un órgano colegiado que tiene, ya de por sí, una serie de demandas institucionales. Por ende, se requiere de una plataforma que destine recursos específicos a la búsqueda de información y a la producción del conocimiento. Por consiguiente, la estrategia implementada fue un proyecto de investigación, que permitió dar respuesta a distintas necesidades desde la alianza de la Escuela con el Instituto de Investigaciones en Educación (INIE), espacio académico en el cual se inscribió.

De esta forma se elabora el proyecto de investigación titulado "Permanencia y graduación de la población estudiantil de Trabajo Social”, cuyo objetivo general plantea: "Proponer un modelo de seguimiento de la permanencia y graduación de la población estudiantil de la Escuela de Trabajo Social de la Sede Rodrigo Facio de la Universidad de Costa Rica que permita identificar situaciones críticas y proponer posibles soluciones" (Regueyra, 2010a, p. 7).

\section{La investigación como estrategia para el mejoramiento}

Como se dijo anteriormente, el proyecto de investigación toma fuerza como estrategia desde la autoevaluación para la reacreditación, en el marco de la cual la Unidad Académica reflexiona sobre la situación del estudiantado -según establece la guía del año 2000 del 
SINAES-, vinculando los temas que han sido neurálgicos y que hasta ese momento no se había logrado resolver. Esto fue posible a través de la discusión en diversos espacios, con docentes, personal administrativo y estudiantes.

Una vez aprobada la reacreditación, se dio inicio al cumplimiento de compromisos para la mejora continua, generados durante la autoevaluación. Estos compromisos deben pensarse vinculados a estrategias sostenibles, que permitan cubrir varias necesidades y responder a su vez a un cúmulo de interrogantes. Elaborar proyectos que estén sostenidos en la plataforma institucional y que puedan movilizar recursos para su cumplimiento permite asumir, de forma estratégica y acertada, la lógica del mejoramiento. Por su parte, el estudio en cuestión permitió cumplir con varios compromisos de mejora desde la plataforma que existe en la Universidad, a partir de una actividad sustantiva -la investigación-, utilizando recursos propios y de un centro de investigación (el INIE). Se logró trabajar de forma sostenida porque se optó por una estrategia acorde con las posibilidades y que respondiera a varias necesidades a la vez.

El estudio también permitió identificar características y necesidades de la población estudiantil, razón de ser de la Universidad y de la Unidad Académica.

Cabe recalcar la enorme responsabilidad que representa formar profesionales que posteriormente tendrán a su cargo, entre otros, el diseño, ejecución y evaluación de políticas sociales, orientadas a poblaciones enfrentadas a diversas desigualdades sociales, como es el caso de Trabajo Social. El proceso educativo, como acto político, debe entonces partir del conocimiento de la población estudiantil, con el fin de mejorar su formación, porque esto impacta necesariamente en el contexto social.

Se sostiene, por lo tanto, que la mejora continua pierde sentido si no se engarza con las necesidades de quienes están en proceso de formación, ya que la calidad y las condiciones de la experiencia educativa impactan las poblaciones meta del Trabajo Social.

Para comprender con mayor claridad la investigación, seguidamente se presenta una síntesis de las elaboraciones conceptuales sobre el tema de deserción y permanencia en el proyecto, el tipo de estudio y el proceso metodológico utilizado, para, después, describir los principales resultados. 


\section{Reflexiones conceptuales y metodológicas}

En el proyecto de investigación fue fundamental entender profundamente el fenómeno educativo denominado "deserción", con el fin de atender sus principales características en el ámbito de la educación superior.

Para estos fines, se consultaron los Estados de la Educación Costarricense, en los cuales se da cuenta de la deserción ligada a la educación secundaria. En estos documentos, se encuentran algunos cuestionamientos al término "deserción" y se alude a la expulsión o exclusión de las y los jóvenes del sistema educativo (Programa Estado de la Nación, 2005 y 2008).

Por otra parte, se revisaron estudios y publicaciones sobre el tema en universidades públicas, tales como los realizados en la Universidad de Costa Rica por Abarca, Jiménez y Sánchez (2003), por Abarca y Sánchez (2008) y la investigación de Castillo (2008) en la Universidad Estatal a Distancia. En estos documentos, se logra visualizar la importancia de este fenómeno en la educación superior, los índices de deserción para cohortes específicas y la necesidad de caracterizar a la población estudiantil.

Asimismo, se revisaron investigaciones a nivel latinoamericano en las que se estudia la deserción en poblaciones universitarias y en diferentes países como Argentina: Giovagnoli P. (2002); Chile: Canales A. y de los Ríos D. (2007) y Himmel E. (2009); México: Rodríguez J. y Hernández J. M. (2008); Uruguay: Boado M. (s.f.) y Colombia: Castaño E., Gallón S., Gómez K. y Vásquez J. (2004); entre otros.

Los resultados de estos estudios aportan que la deserción se refiere a la población estudiantil que ingresó a una carrera, no continúa matriculando y no logra la titulación. Esta definición general adquiere otras especificidades si esta situación obedeció a una decisión del estudiante (deserción voluntaria) o a una baja reglamentaria (deserción involuntaria). Otros criterios que se han considerado en las definiciones son: primero, el tiempo en que sucede el evento, es decir, si se presenta en el proceso de admisión, durante la carrera, en uno o varios ciclos electivos; y el espacio, o lugar de la deserción: si es de una carrera, de un plan de estudios, de una universidad o del sistema educativo en general.

En relación con las definiciones sobre deserción, se plantea que cada investigador o investigadora define la que aplicará, al respecto: "...se afirma que ninguna definición puede captar en su totalidad la complejidad de este fenómeno, quedando en manos de los 
investigadores la elección de la definición que mejor se ajuste a sus objetivos y al problema a investigar" (Castaño, Gallón, Gómez y Vásquez, 2004, p. 45).

En las investigaciones mencionadas se utilizan diversas metodologías, entre las cuales están: estudio descriptivo, análisis de transición o análisis de duración, análisis multivariado, estudio cualitativo exploratorio y otros para identificar índices o probabilidades de deserción. Además, se caracteriza demográfica y académicamente a la población que deserta y sus motivos.

En cuanto a los modelos para explicar la deserción, los estudios recuperan los psicológicos, sociológicos, económicos, organizacionales e integrales.

Himmel elabora un resumen de los diversos enfoques teóricos utilizados en estudios de deserción en los últimos 25 años y señala la importancia de identificar cómo se combinan las distintas variables en diferentes tipos y modalidades institucionales. Indica al respecto:

(...) como síntesis de los modelos expuestos, se puede señalar que éstos incluyen diversas dimensiones o combinaciones de las mismas. Se ha logrado determinar también la medida en que dichos factores promueven o inhiben la deserción estudiantil en la educación superior. Parece necesario, entonces, indagar sistemáticamente estos lineamientos en el país, a fin de identificar cómo se combinan las distintas variables en los diferentes tipos y modalidades institucionales, de modo que éstas puedan actuar sobre los factores que son más controlables por las propias instituciones y así reducir el costo social económico que conlleva la deserción. (Himmel, 2009, p. 108)

La revisión de estas investigaciones favoreció la reflexión sobre el objeto de investigación y permitió concluir que:

1. La deserción se refiere a aquellas personas que no continúan matriculando en la Universidad o la carrera en estudio en un periodo definido.

2. El concepto de deserción es solo parte de un fenómeno más complejo: la permanencia de la población estudiantil.

3. Investigar la permanencia de la población estudiantil permite una comprensión más amplia si considera esta como:

(...) el proceso macro que inician los y las estudiantes desde la etapa de ingreso a la universidad, que continúa con el proceso de permanencia en la carrera, hasta la 
obtención de un título universitario. Es por lo tanto el proceso que vive la población estudiantil desde su primer contacto con la Universidad hasta su graduación. (Regueyra y Rojas, 2011a, p. 11)

4. No existe un único modelo de referencia a seguir para estudiar este fenómeno. Depende de las condiciones institucionales y del contexto.

5. Resulta más enriquecedor contar con la mayor cantidad de variables y elementos posibles para buscar una comprensión más completa.

6. El análisis de las metodologías para estudiar la deserción, permitió comprender lo acertado de acercarse al objeto mediante un estudio de cohortes, es decir, grupos de estudiantes que ingresaron en un año específico y a quienes se da seguimiento a lo largo del tiempo para recabar datos relevantes.

Además de lo mencionado, durante el proceso de investigación se construyó una serie de productos que se mencionan en el siguiente apartado, en el cual se explicita el tipo de investigación y el proceso seguido.

\section{Tipo de investigación y proceso metodológico}

La investigación se planteó como un estudio descriptivo que permitió un primer acercamiento a la permanencia de la población estudiantil de Trabajo Social. En términos generales, los estudios descriptivos pretenden:

....medir o recoger información de manera independiente o conjunta sobre concepto o las variables a los que se refieren. Desde luego, pueden integrar las mediciones o información de cada una de dichas variables o conceptos para decir cómo es y cómo se manifiesta el fenómeno de interés; su objetivo no es indicar cómo se relacionan las variables medidas. (Hernández, Fernández y Batista, 2003, p.119)

Lo anterior se cumplió mediante un estudio de cohortes estudiantiles, considerando una cohorte como "la población estudiantil que ingresó a la carrera de Trabajo Social en un año específico y concretó la matrícula" (Regueyra, 2010b, p. 60).

El estudio de cohortes permite seguir longitudinalmente a una o varias poblaciones, por

lo que favorece identificar la temporalidad de los sucesos vinculados con el objeto de 
estudio, en este caso, la permanencia de la población estudiantil en la carrera de Trabajo Social de la Universidad de Costa Rica, de cada una de las cohortes de 2002 a 2009.

En la investigación, el acercamiento al objeto se realizó combinando estrategias cualitativas y cuantitativas, como se describe a continuación, en una síntesis del proceso metodológico utilizado:

1. Búsqueda y análisis bibliográfico: en primera instancia se realizó una búsqueda de documentos que dieran cuenta de investigaciones similares a nivel nacional e internacional, tal como se describió en el apartado anterior. Esto fue fundamental a nivel conceptual y metodológico y permitió esclarecer la importancia de hacer un estudio de cohortes desde el tema de permanencia -no de deserción-, recuperando para esto definiciones y variables demográficas, académicas y socioeconómicas.

2. Construcción de un sistema de información: dada la experiencia y los conocimientos de la investigadora principal sobre las bases de datos con las que cuenta la Universidad de Costa Rica, se logró determinar que era posible captar la información demográfica, académica y socioeconómica del estudiantado del Sistema de Aplicaciones Estudiantiles (SAE). Dicho Sistema se creó para realizar los trámites de admisión, matrícula, cobro y becas de toda la Universidad, por lo cual cuenta con gran cantidad de información sobre el estudiantado, que se utiliza únicamente con fines administrativos y para generar algunas estadísticas básicas de cada una de las oficinas encargadas de estos procesos. Se valoró que era posible capturar la información, para lo cual se tuvo que elaborar otra serie de aplicaciones informatizadas, mediante el apoyo de una profesional en Informática. Para esto, las investigadoras definieron una serie de preguntas, hipótesis y variables que serían la base para la construcción del sistema.

Las interrogantes se orientaron a determinar el porcentaje de población estudiantil de Trabajo Social de las cohortes de 2002 a 2009 que cumple con el plan estudio, si este se retrasa o no continúa, así como las características demográficas, socioeconómicas y académicas que influyen en que la población estudiantil cumpla con el plan estudio, se retrase o no continúe.

Las hipótesis fueron una guía para trabajar, posteriormente, el procesamiento estadístico de la información generada. Sus resultados se exponen más adelante. 
Las variables definidas que permitieron estudiar y captar la permanencia, a partir de los datos extraídos del SAE, se presentan en la Tabla 1.

Tabla 1

Variables para captar la permanencia

\begin{tabular}{|c|c|c|c|c|}
\hline \multirow[t]{2}{*}{ Variables } & \multirow[t]{2}{*}{ Demográficas } & \multicolumn{2}{|c|}{ Académicas } & \multirow[t]{2}{*}{ Socioeconómicas } \\
\hline & & Admisión & Permanencia & \\
\hline \multirow{5}{*}{$\begin{array}{l}\frac{\mathscr{d}}{0} \\
\frac{0}{\pi} \\
\frac{\pi}{\pi} \\
>\end{array}$} & Edad & $\begin{array}{c}\text { Prueba de aptitud } \\
\text { académica }\end{array}$ & Carga académica & Tipo de beca \\
\hline & $\begin{array}{l}\text { Edad ingreso } \\
\text { UCR y a } \\
\text { Trabajo Social }\end{array}$ & Nota de admisión & Rendimiento & Categoría de beca \\
\hline & Sexo & Matrícula & Grado académico & $\begin{array}{c}\text { Beneficios } \\
\text { complementarios }\end{array}$ \\
\hline & Procedencia & Opción de carrera & \multirow[t]{2}{*}{ Carreras cursadas } & \multirow[t]{2}{*}{ Ayuda económica } \\
\hline & Tipo de colegio & $\begin{array}{l}\text { Modalidad de } \\
\text { ingreso }\end{array}$ & & \\
\hline
\end{tabular}

Fuente: Elaboración propia, 2012

Una vez definidas estas variables, se realizó un largo proceso de construcción de las nuevas aplicaciones que permitió extraer la información del SAE. Esto implicó una minuciosa revisión y depuración de los datos que eran extraídos mediante las aplicaciones. El conjunto de aplicaciones se denominó Sistema de Seguimiento a la Permanencia de la Población Estudiantil (en adelante SISEPPOE). Dicho sistema permitió migrar hacia Excel la información del SAE y crear una base de datos con el historial académico de cada estudiante, desde su ingreso a la Universidad hasta que deja de matricular. Esta base de datos fue denominada "Tabla de vida académica" y contempló la información de las y los estudiantes que ingresaron a la carrera y consolidaron su matrícula (es decir, que matricularon al menos un curso del plan de estudio), desde el 2002 hasta el 2009 (Regueyra y Rojas, 2011b).

3. Procesamiento estadístico de la información: los datos que se generaron en la "Tabla de vida académica" fueron procesados estadísticamente para lograr evidenciar las características demográficas, académicas y socioeconómicas que explican la permanencia del estudiantado en la carrera y sus tiempos de graduación. Se trabajó en conjunto con una profesional de estadística, para quien las hipótesis representaron una guía para organizar la información. 
4. Caracterización de la población estudiantil: a partir de lo aportado en la "Tabla de vida académica" y en el análisis estadístico, fue posible caracterizar a la población estudiantil de las cohortes que fueron parte del estudio. Esto permitió dar respuesta o bien rechazar las hipótesis, conocer las particularidades que presenta la población de estudiantes de la carrera en el periodo de estudio, así como conocer cómo se manifiesta la permanencia y cuáles estudiantes continúan la carrera y se gradúan, en cuánto tiempo lo hacen y cuáles no continúan.

5. ¿Qué pasa con quienes no continúan? Posterior a lo descrito, fue posible contactar a una buena parte del estudiantado que no continúa y consultar, mediante entrevista telefónica, las razones por las cuales dejaron la carrera.

6. Identificación de situaciones críticas: con la caracterización lista, incluida la información de quienes no continúan, se desarrolló un grupo focal con docentes, personal administrativo y estudiantes de la Unidad Académica. En este espacio, se compartieron los resultados del proyecto, se entregó un documento para la identificación de situaciones críticas que cada participante fue completando individualmente y, al final, se reflexionó sobre cuáles eran los temas neurálgicos evidenciados por el estudio. Esta parte cualitativa del proyecto permitió compartir resultados y generar un proceso participativo de reflexión, para alimentar la toma de decisiones.

El proceso descrito en este apartado permite captar cómo el proyecto fue generando una serie de resultados, los cuales se exponen en el siguiente punto.

\section{Resultados}

La información de la población estudiantil de Trabajo Social se obtuvo del Sistema de Aplicaciones Estudiantiles (SAE), aplicación que permite a la Universidad de Costa Rica almacenar la información de la población estudiantil generada desde los diversos procesos de admisión, matrícula, graduación, becas y beneficios complementarios, entre otros. El proyecto, a su vez, desarrolló aplicaciones que permitieron capturar la información que se requería y presentarla en una tabla en Excel (aspectos que se detallaron en el apartado anterior). Esto permitió dar seguimiento a la permanencia de la población estudiantil de manera individual, por cohorte y población total matriculada en la escuela de Trabajo Social en el periodo 2002 al 2008, para un total de 385 estudiantes y 7 cohortes estudiantiles. 
A continuación, se comparten los resultados del procesamiento de la información que se agrupan para dar respuesta a las siguientes interrogantes:

- ¿Quiénes son las y los estudiantes?

- ¿Cómo es la permanencia y graduación de la población estudiantil de Trabajo Social?

- ¿Quién es la población estudiantil que no continúa?

\section{1. ¿Quiénes son las y los estudiantes?}

La población estudiantil es predominantemente femenina: la cantidad de estudiantes de dicho sexo supera el $80 \%$ en las 7 cohortes, con una edad de ingreso entre 17 y 20 años, seguido de quienes ingresan de 21 o 22 años. Menos del 10\% de la población ingresa con más de 22 años.

La mayor parte de la población que ingresa a la carrera procede de la Gran Área Metropolitana y casi un $17 \%$ procede de zonas consideradas como alejadas del centro de estudios. Esto obliga a las y los estudiantes a trasladarse desde sus zonas de procedencia para vivir en lugares más cercanos al centro de estudios.

El comportamiento de cada una de las cohortes en estudio se puede observar en el gráfico 1:

Gráfico 1. Población estudiantil de la Escuela de Trabajo Social por zona de procedencia y cohorte, 2002-2006

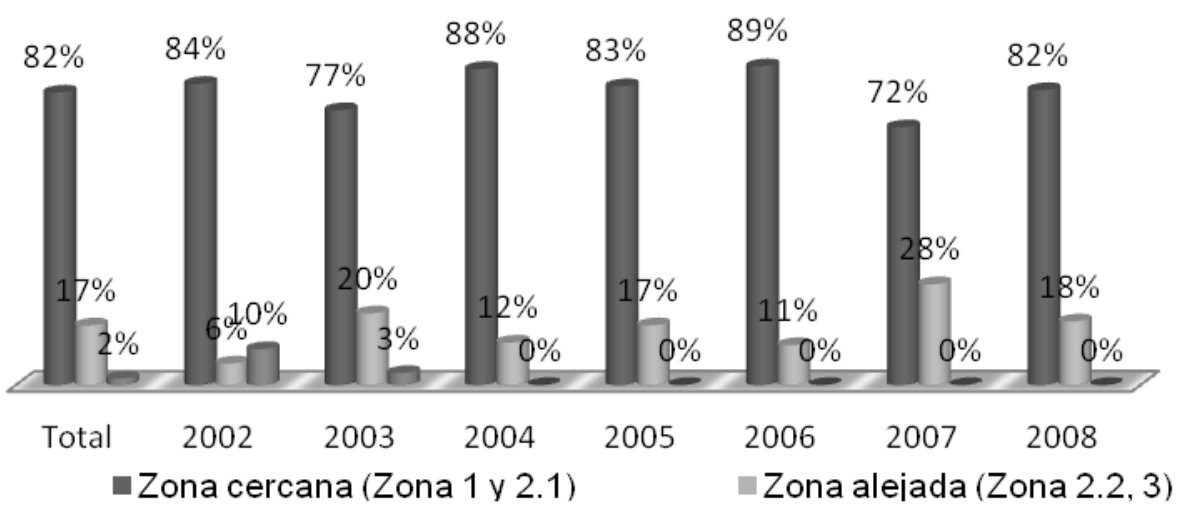

Fuente: Elaboración propia, Proyecto "La Permanencia y graduación de la población estudiantil de Trabajo Social de la Universidad de Costa Rica", 2011 
En relación con el colegio de procedencia, se encontró que el $66 \%$ de la población estudiantil realizó sus estudios en instituciones públicas. El $84 \%$ de los colegios de procedencia de la población estudiantil poseen modalidad académica.

La mayoría de la población que ingresó a la carrera en el periodo de estudio lo hizo mediante la Prueba de Aptitud Académica (PAA) ${ }^{3}$, ya sea que ingresó directamente a la carrera o que se trasladó de otra carrera a Trabajo Social. Los puntajes obtenidos por esta población estudiantil en la PAA se ubican en mayor porcentaje en el rango de 550 a 599 y en el rango de 600 a 649.

Un 73\% de la población ha estado empadronada solo en Trabajo Social, sin embargo, 138 estudiantes han estado empadronados en dos carreras, 19 estudiantes en 3 carreras y 6 estudiantes en 4 carreras, en la Universidad de Costa Rica.

La gran mayoría de la población estudiantil, un $87 \%$, ha disfrutado de algún tipo de beca (Beca de Honor, de Estímulo, de Excelencia o por Condición Socioeconómica) al menos durante un ciclo lectivo, sin embargo, cada cohorte tiene un comportamiento diferente, como se puede apreciar en el gráfico 2 .

\footnotetext{
${ }^{3}$ El puntaje máximo es de 800 puntos.
} 
Gráfico 2. Población estudiantil de la Escuela de Trabajo Social por beca

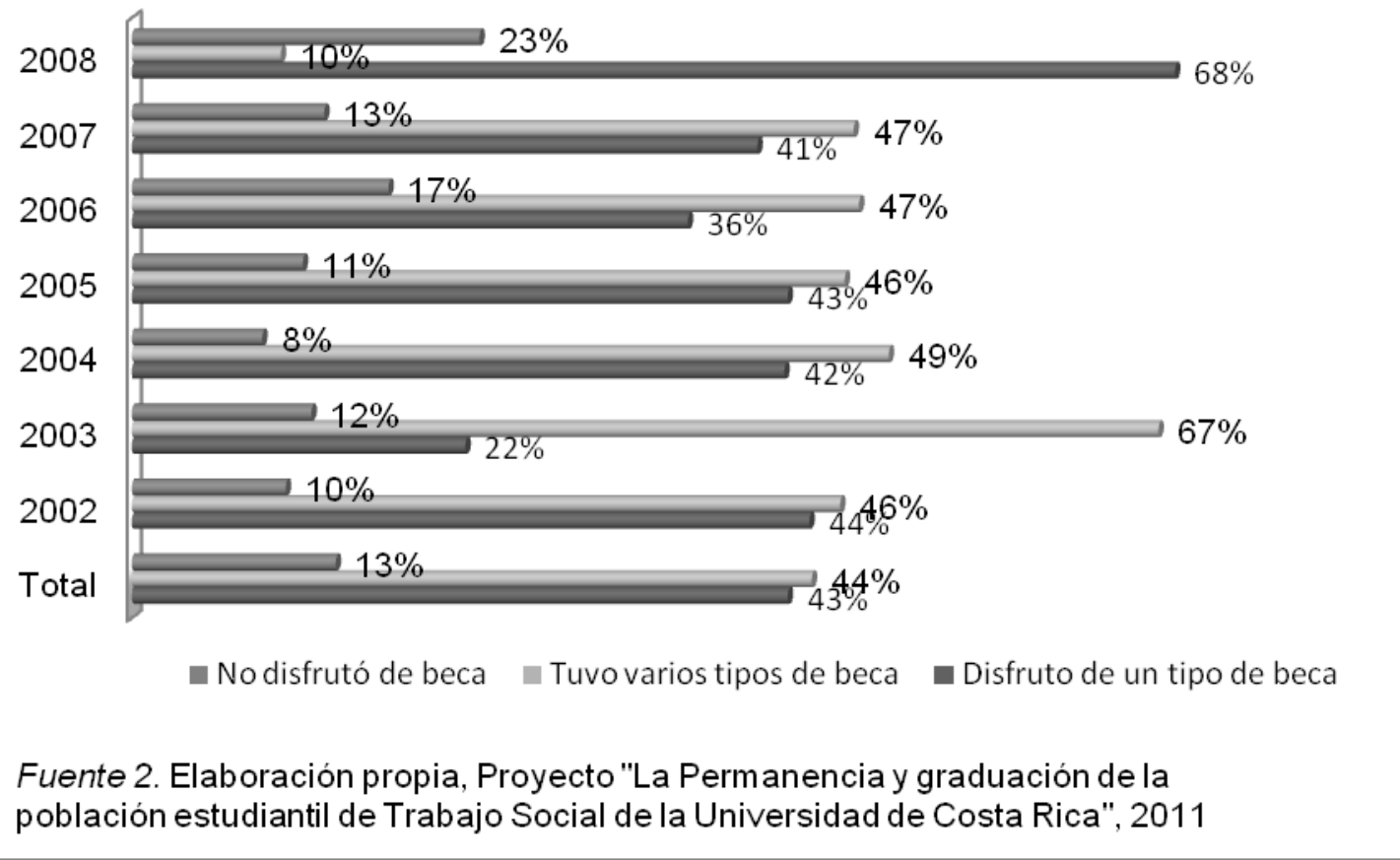

Del total de población que ha disfrutado de beca, el $76,7 \%$ ha tenido beca por condición socioeconómica. Es significativo que gran cantidad de estudiantes que han disfrutado de esta beca ha recibido beneficios complementarios y ayuda económica de la Universidad, estos se otorgan a la población que procede de familias con limitados recursos económicos de acuerdo con el índice socioeconómico definido por la Universidad de Costa Rica.

La población estudiantil matricula en los I y II ciclos lectivos un promedio 12,8 créditos, con una moda de 17 créditos, considerando solo los cursos correspondientes a su plan de estudio. Por otra parte, si además se toman en cuenta los cursos que no son de su plan de estudio en Trabajo Social, el promedio es de 16,7 créditos, con una moda de 18 créditos. La población estudiantil aprueba 12 créditos en promedio en los I y II ciclos lectivos con una moda de 17 créditos aprobados, el $34 \%$ de la población aprueba 15 créditos o más, la variación se puede observar en el gráfico 3. 
Gráfico 3. Población estudiantil de la Escuela de Trabajo Social, por total de créditos aprobados en Trabajo Social (I y II ciclo) y cohorte, 2002-2008

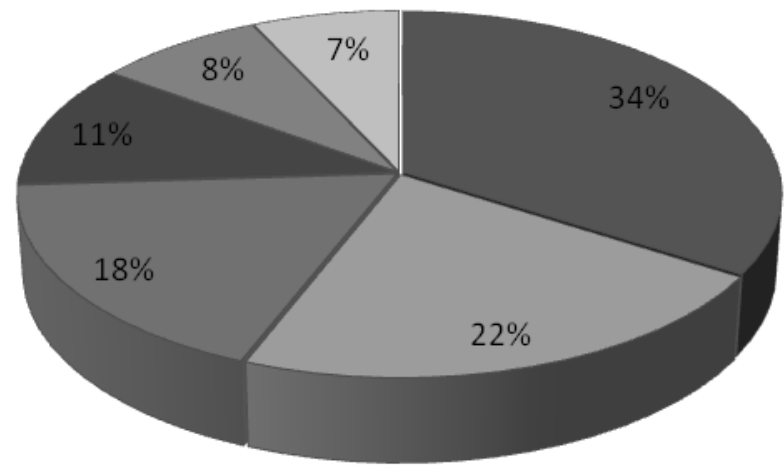

15 creditos o más

口Entre 12 y menos de 14 créditos

$\square$ Menos de 10 créditos

Entre 14 y menos de 15 créditos

$\square$ Entre 10 y menos de 12 créditos

Fuente: Elaboración propia, Proyecto "La Permanencia y graduación de la población estudiantil de Trabajo Social de la Universidad de Costa Rica", 2011.

El total de créditos matriculados del plan de estudio, por ciclo lectivo, es una variable que afecta la permanencia de la población estudiantil, especialmente aquella población que matricula menos número de créditos que lo estipulado en el plan de estudio de Trabajo Social para cada ciclo lectivo.

Cerca de una cuarta parte $(23,6 \%)$ de la población estudiantil de Trabajo Social presenta promedio ponderado insuficiente (notas menores a 7 ) en cursos que pertenecen al plan de estudio en Trabajo Social; un 76,4\% presenta promedios ponderados superiores a 7. Por otro lado, dos terceras partes (66\%) se ubican en promedios entre bueno y muy bueno $(7,4$ y 9,4). Casi la mitad de la población estudiantil de Trabajo Social ha perdido créditos alguna vez y son 3 los créditos que pierde con mayor frecuencia.

El $87,7 \%$ de la población de Trabajo Social, considerando todos los créditos matriculados en la Universidad, tiene promedios ponderados superiores a 7 (nota mínima para aprobar un curso) y sólo un 12,3\% tiene promedios ponderados insuficientes. 


\section{2. ¿Cómo es la permanencia y graduación?}

Sobre la permanencia de la población estudiantil de Trabajo Social de las cohortes 2002 a 2006 (que son las poblaciones que hasta el año 2009 tienen cuatro o más años de haber consolidado su ingreso a Trabajo Social), un 43,2\% tiene una permanencia de cuatro años para alcanzar el título de Bachillerato en Trabajo Social y un 19\% lo logra en más de 4 años. En el gráfico 4 se detalla el comportamiento de las cohortes 2002 al 2006.

Gráfico 4. Población estudiantil de la Escuela de Trabajo Social por estatus en Bachillerato y cohorte, 2002-2006

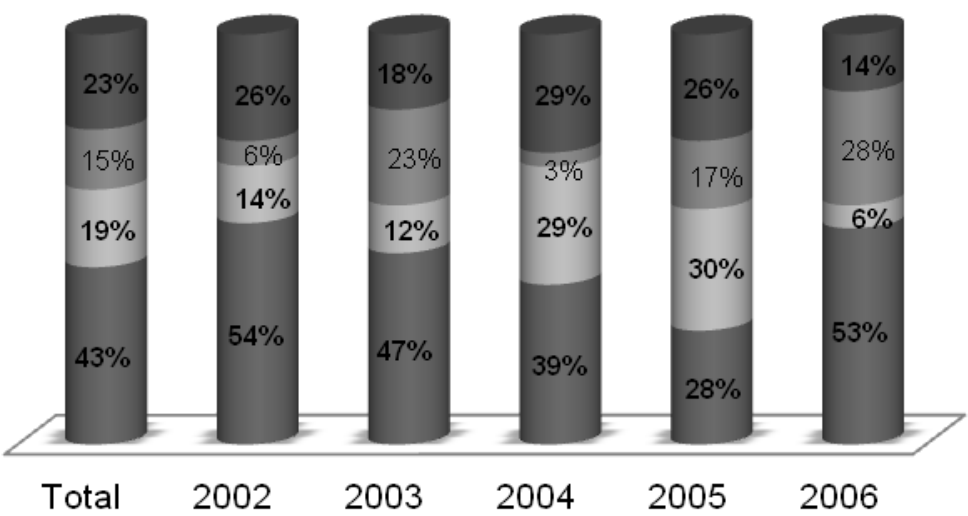

- Terminan el bachillerato en 4 años

- Terminan el bachillerato en más de 4 años

- No han terminado

- No continúan matriculando (abandonan)

Fuente: Elaboración propia, Proyecto "La Permanencia y graduación de la población estudiantil de Trabajo Social de la Universidad de Costa Rica", 2011.

Nota: El total de la población de las cohortes 2002 al 2006 es de 259 estudiantes.

En relación con el periodo para obtener la Licenciatura, considerando las cohortes 2002 a 2004 (que son las poblaciones que hasta el año 2009 tienen seis o más años de haber consolidado su ingreso a Trabajo Social), un 31,4\% tienen una permanencia de seis años para alcanzar el título de Licenciatura en Trabajo Social y un 15\% lo logra en más de 6 años. En el gráfico 5 se puede apreciar el comportamiento de cada cohorte en estudio, llama la atención que la cohorte del 2004 presenta un 39\% de población egresada (aprobó todas las materia del plan de estudio), que no ha obtenido el título de Licenciatura por tener pendiente su Trabajo Final de Graduación. 
Gráfico 5. Población estudiantil de la Escuela de Trabajo Social por estatus en Licenciaturay cohorte, 2002-2006

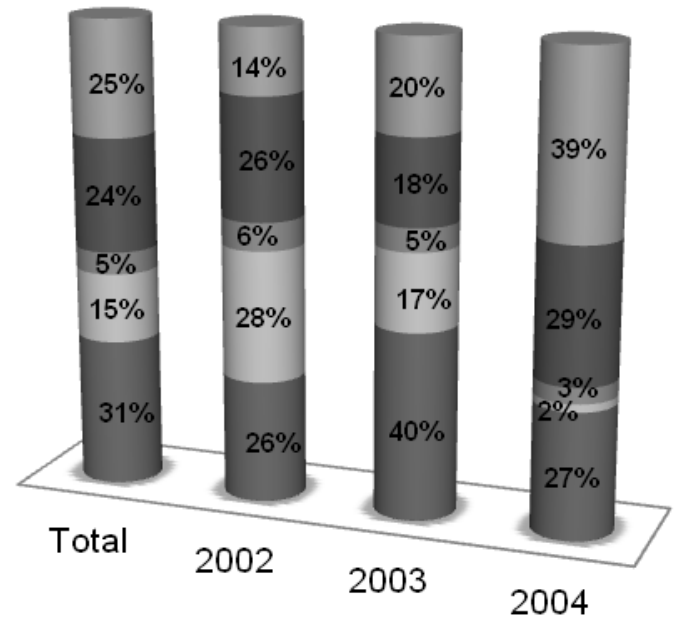

- Termina a tiempo según plan

- Termina pero no a tiempo

- No termina pero sigue matriculando

- No continúa matriculando (abandonan)

Fuente: Elaboración propia, Proyecto "La Permanencia y graduación de la población estudiantil de Trabajo Social de la Universidad de Costa Rica", 2011 Nota: El total de la población de las cohortes 2002 al 2004 es de 169 estudiantes

El haber ingresado a la carrera de Trabajo Social el mismo año en que ingresó a la Universidad de Costa Rica, el tener edades entre los 17 y los 18 años, el proceder de zonas cercanas a la Sede Rodrigo Facio, el haber realizado estudios en colegios privados o semipúblicos en la modalidad académica, el haber disfrutado de becas y beneficios complementarios, el matricular cursos del plan de estudio en Trabajo Social, el matricular más de 15 créditos por ciclo lectivo con promedios ponderados superiores a 7 , el haber aprobado créditos en el tercer ciclo lectivo y no haber perdido créditos, son aspectos que se presentan en porcentajes más altos en la población estudiantil que tiene una permanencia de 4 años para la obtención del título de Bachillerato y de 6 años para obtener el título de Licenciatura.

\section{3. ¿Quién es la población que no continúa?}

El 22\% de la población de las cohortes 2002 a 2008 no continúa matriculando cursos en la carrera de Trabajo Social, esto corresponde a un total de 86 estudiantes. La cohorte 2004 presenta los porcentaje más altos de no continuación, seguida de las cohortes 2003 y 
2005, como se detalla en el gráfico 6. Dos cuartas partes no continúan a partir del segundo ciclo lectivo y una cuarta parte a partir del tercer y cuarto ciclos lectivos.

Gráfico 6. Cantidad total de estudiantes que no continúan (dejan de matricular), según cohorte del 2002-2008

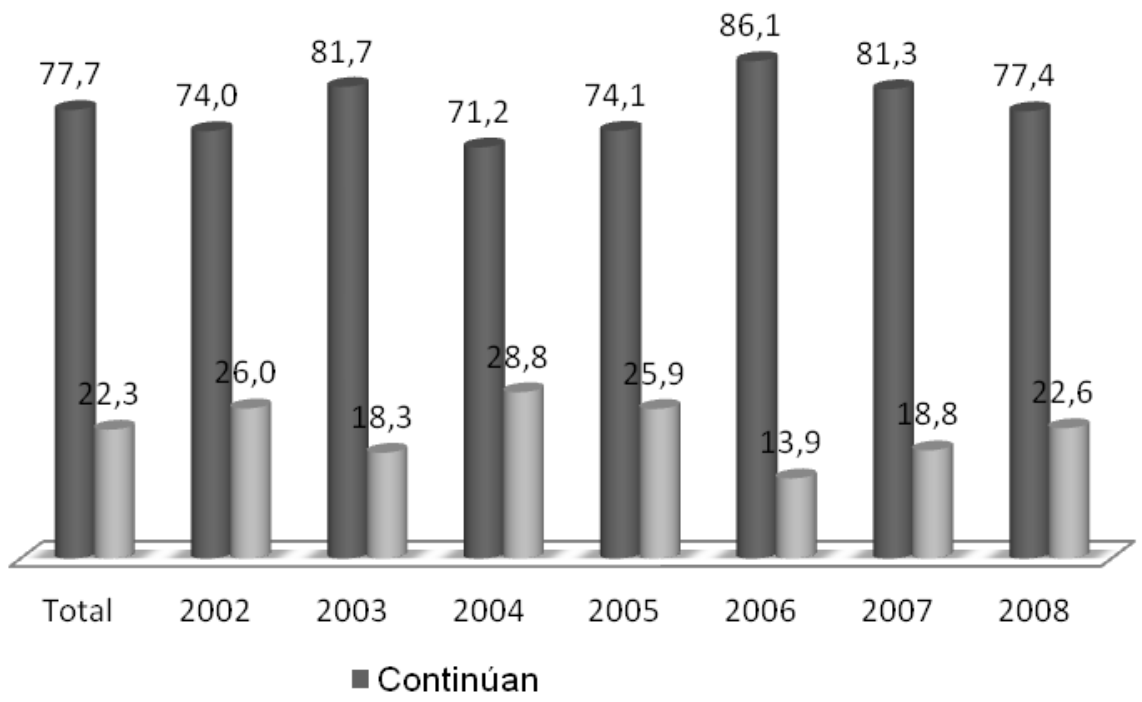

Fuente: Elaboración propia, Proyecto "La Permanencia y graduación de la población estudiantil de Trabajo Social de la Universidad de Costa Rica", 2011.

Del total de estudiantes que no continúa, se logró entrevistar al 50\%, esto es un total de 43 estudiantes. De estos, el 58,14\% manifiesta que la razón principal por la que no continuó es el interés hacia otra carrera; en segundo lugar, que el horario de clases no les permitía trabajar y estudiar. Esta información se detalla en el gráfico 7: 
Gráfico 7. Población estudiantil entrevistada que no continúa en la Escuela de Trabajo Social ,según motivos de no continuación, 2002-2009
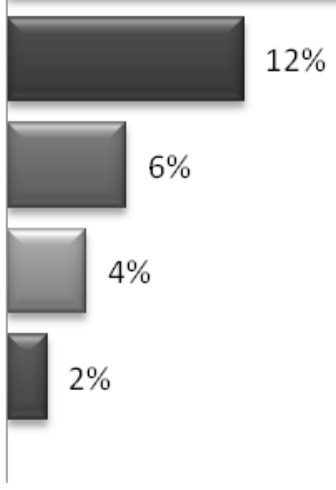

\author{
$\triangle$ Interés en otra carrera \\ - Horario no permite trabajar y estudiar \\ - Situación económica \\ $\square$ Otros \\ $\square$ Situación con la Universidad o la carrera \\ घSituación personal
}

Fuente: Elaboración propia, Proyecto "La Permanencia y graduación de la población estudiantil de Trabajo Social de la Universidad de Costa Rica", 2011.

El $83,72 \%$ de la población entrevistada continúa estudiando, un 65,85\% en universidades privadas y un $21,95 \%$ en la Universidad de Costa Rica. Al referirse a su experiencia en la Escuela de Trabajo Social, para algunas personas, esta fue positiva: "me gustó mucho por la parte social pero en ese momento yo trabajaba", "fue muy bonita la carrera es muy completa, aunque usted sabe que en el fondo uno no puede estudiar algo que no le guste", "aprendí a estudiar"; otras personas la consideraron negativa: "Darme cuenta que es el trabajo más frustrante del mundo, tanto a nivel académico como en lo se puede hacer a nivel profesional", "Nunca fue buena, yo estuve año y medio y el ambiente fue muy chocante". Por otra parte, entre las apreciaciones de la población estudiantil que no continúa se identificaron disconformidades con los contenidos y estrategias didácticas en los 
primeros cursos de la carrera, a modo de ejemplo, los siguientes comentarios: "Me encantó lastimosamente llevé los cursos feos de primer año" "yo creo que el plan de estudios estaba bien, pero era necesario hacer las clases más dinámicas".

Entre las sugerencias que se hacen a la Unidad Académica están las siguientes: ofrecer horarios flexibles, que la población docente realice un mayor acercamiento con la población estudiantil en actividades de comunicación y apertura hacia sus necesidades, tomando en cuenta que son estudiantes de primer año, es decir, que experimentan la transición del colegio a la universidad.

\section{Conclusiones}

Se desarrolló un estudio descriptivo sobre la permanencia de la población estudiantil de Trabajo Social, el cual aportó información para describir el objeto de investigación, utilizando un análisis básico a nivel estadístico que puede ser la plataforma para futuras aproximaciones de carácter analítico.

La investigación combinó estrategias cualitativas y cuantitativas, lo que favoreció el acercamiento al objeto de investigación, desde la información almacenada en el Sistema de Aplicaciones Estudiantiles así como desde la visión de estudiantes, docentes y administrativos, lo que permitió la articulación de ambas posibilidades.

La consulta de investigaciones sobre deserción en otros contextos favoreció la reflexión del equipo investigador, el cual asumió la permanencia como objeto de investigación, considerándola como el proceso macro que vive la población estudiantil desde la etapa de ingreso a la Universidad, hasta la obtención de un título universitario. La deserción fue asumida como una parte de ese proceso integral que vive la población estudiantil, desde su primer contacto con la Universidad, hasta la graduación.

En relación con la población estudiantil de Trabajo Social, se puede afirmar que de las cohortes 2002 al 2008: un 43.2\% permanece 4 años para obtener el título de Bachiller en Trabajo Social mientras que en un 31.4\%, la permanencia es de 6 años para obtener el título de Licenciatura.

La investigación permitió identificar que la población estudiantil de Trabajo Social de las cohortes 2002 al 2008 que tienen una permanencia de 4 años para la obtención del título de Bachillerato y 6 años para obtener el título de Licenciatura, presentan porcentajes más altos en los siguientes aspectos: haber ingresado el mismo año a la Universidad de Costa 
Rica y a la carrera de Trabajo Social, tener edades entre los 17 y los 18 años, proceder de zonas cercanas a la Sede Rodrigo Facio, haber realizado estudios en colegios privados o semi-públicos en la modalidad académica, haber disfrutado de becas y beneficios complementarios, matricular solamente cursos del plan de estudios en Trabajo Social, matricular más de 15 créditos por ciclo lectivo con promedios ponderados superiores a 7 , haber aprobado créditos en el tercer ciclo lectivo y no haber perdido créditos.

En el nivel de Licenciatura, la mayoría de la población estudiantil permanece más de 6 años para obtener el título, lo cual evidencia que hay estudiantes que ya han concluido con los cursos del plan de estudios, es decir son egresados o egresadas, pero aún no logran graduarse. Por lo que se puede afirmar que tienen pendiente el Trabajo Final de Graduación.

La población estudiantil de Trabajo Social, en un alto porcentaje, procede de hogares con limitaciones económicas y, a pesar de que el 76,7\% ha disfrutado del Sistema de Becas por Condición Socioeconómica de la Universidad, que incluye exoneración sobre los montos de matrícula, ayuda económica y beneficios complementarios, esto es un apoyo que no les permite satisfacer la totalidad de sus necesidades socioeconómicas. Por lo anterior, las y los estudiantes deben complementar con otro tipo de actividades para lograr ingresos económicos, tales como las horas estudiante, horas asistente, o laborar por jornadas parciales y temporales fuera de la Universidad. Algunas veces, no continúan con los estudios, como en las situaciones particulares que se identificaron en las entrevistas realizadas a esta población. En este sentido, cabe señalar que la Escuela de Trabajo Social no ofrece una modalidad nocturna, ni de fines de semana.

La situación anterior se agrava en el caso de la población que procede de zonas alejadas del centro de estudios, ya que requiere de mayores recursos económicos para mantenerse fuera de su hogar de origen. La Universidad de Costa Rica, a pesar de contar con el Programa de Residencias como parte del Sistema de Becas, no logra satisfacer la demanda de toda la población estudiantil que requiere de este servicio y que se encuentra cursando el nivel de Bachillerato. Además, este beneficio no cubre a la población que cursa Licenciatura. Los efectos de esta situación se evidencian cuando se observan los porcentajes de obtención del título de Bachillerato: la población estudiantil que procede de lugares alejados presenta un porcentaje menor al de quienes proceden de lugares cercanos al centro de estudios, en cuanto a lograr el Bachillerato en 4 años. 
El tipo de colegio del que proceden las y los estudiantes (público, privado o semipúblico) y la modalidad (académica, técnica, artística, abierta entre otras) es una categoría que presenta comportamiento diverso en relación con la graduación en 4 años para bachillerato; la población que procede de colegios privados o semipúblicos tiene un mayor porcentaje de graduación en 4 años que la procede de colegios públicos. Los que proceden de colegios con modalidad académica presentan más altos porcentajes en graduarse en 4 años que quienes proceden de colegios públicos, con modalidades técnicas, artísticas y modalidad abierta.

Las situaciones socioeconómicas, la ubicación geográfica de la familia y la modalidad y tipo de colegio de procedencia, descritas en los párrafos anteriores, son características socio-demográficas de la población estudiantil de Trabajo Social que influyen en los periodos más largos de permanencia para lograr el título de Bachillerato o Licenciatura

Un $22 \%$ de la población de las cohortes no continúa matriculando cursos del plan de estudio de la carrera de Trabajo Social. El porcentaje de estudiantes que no continúa en la carrera se relaciona directamente con el haber ingresado a una carrera que no era de su interés, lo que se evidencia en la opinión que les generan los primeros cursos de la carrera como "des-motivantes" por su contenido, enfoque, vocabulario y estrategia didáctica. Lo anterior sin descartar que lo señalado tenga relación con la propuesta programática de los cursos del primer año. El bajo rendimiento académico de la población estudiantil que no continúa es una característica de esta población y, al mismo tiempo, un aspecto que debe alertar a la Unidad Académica.

\section{Recomendaciones}

A. Compartir con la población docente, estudiantil y administrativa de la Escuela de Trabajo Social los resultados de esta investigación así como la propuesta de dar seguimiento a la población estudiantil de Trabajo Social desde las variables que aporta este estudio.

B. Realizar capacitaciones acerca de las características de la población estudiantil de Trabajo Social; así como del Sistema de Becas de la Universidad (con detalle de servicios, fechas y requisitos para las solicitudes correspondientes) y sobre la carrera, los contenidos, la organización del plan curricular y las expectativas en los tiempos de 
graduación; dirigidas a la población docente que cumple funciones de consejería y acompañamiento.

C. Elaborar estrategias de acompañamiento y apoyo para estudiantes:

- De primer ingreso, que contemple acciones de información y asesoría sobre la carrera (contenidos, organización del plan de estudio y las expectativas en los tiempos de graduación), así como de los servicios estudiantiles que ofrece Universidad (detalle de servicios, fechas y requisitos para las solicitudes correspondientes).

- Con mayores limitaciones económicas y procedentes de colegios públicos con modalidad técnica, artística y abierta, así como a la población procedente de lugares alejados de la Sede Rodrigo Facio, para favorecer su permanencia y graduación.

D. En el primer año de la carrera.

- Fortalecer los cursos introductorios de primer año, considerando las características de la población de primer ingreso, sus expectativas y el respeto a la diversidad ideológica.

- Implementar estrategias pedagógicas dinámicas, que no sobrecarguen de información a las y los estudiantes y les acerquen al conocimiento de la carrera.

E. En el nivel de licenciatura de la carrera.

- Revisar los factores que afectan la culminación oportuna del Trabajo Final de Graduación, ya que esto provoca que se aumente el tiempo para obtener el título.

- Valorar la incorporación de oferta académica en el III ciclo lectivo de cursos del plan de estudio que permita a la población estudiantil avanzar en la carrera y disminuir la presión en el I y II ciclos lectivos.

F. Que la Escuela de Trabajo Social continúe dando seguimiento a la permanencia de la población estudiantil, para lo cual se propone:

- Consolidar un equipo responsable de realizar esta labor.

- Dar seguimiento a las cohortes estudiantiles investigadas y a las nuevas cohortes que se incorporen a la carrera.

- Dar seguimiento a la población estudiantil que cursa otras carreras, además de Trabajo Social. 
- Desarrollar mecanismos permanentes para conocer las necesidades de la población estudiantil.

- Identificar estudiantes que podrían no continuar en la carrera, considerando situaciones como el bajo rendimiento académico y el número de créditos matriculados y perdidos del plan de estudio.

- Recuperar aspectos positivos sobre la permanencia y el rendimiento de las y los estudiantes de las cohortes que han presentado mejor desempeño académico y mayor porcentaje de graduación en 4 años para Bachillerato y 6 años para la Licenciatura, como es el caso de la cohorte 2006.

\section{Propuestas de futuras investigaciones}

- Indagar sobre los factores que afectan el rendimiento académico desde las particularidades y exigencias propias de la carrera de Trabajo Social.

- Identificar los factores que favorecieron o limitaron la permanencia de la población estudiantil que se graduó en el tiempo estipulado en el plan de estudio, la población que aún permanece en la carrera así como la que se graduó en un periodo mayor, considerando sus preocupaciones, opiniones y experiencias.

\section{Referencias}

Abarca, Allan y Sánchez, María A. (2008). La deserción estudiantil en la educación superior: El caso de la Universidad de Costa Rica. Revista Electrónica Actualidades Investigativas en Educación, 5(4), 1-22. Recuperado de http://revista.inie.ucr.ac.cr/buscar-articulos/controlador/Article/accion/show/articulo/ladesercion-estudiantil-en-la-educacion-superior-el-caso-de-la-universidad-de-costarica.html

Abarca, Allan, Jiménez Jairo y Sánchez, María A. (2003). Deserción en la Universidad de Costa Rica: un estudio de magnitud y característica de los desertores. San José, Costa Rica: Centro de Evaluación Académica Universidad de Costa Rica.

Boado, Marcelo. (s.f.). Una aproximación a la deserción Estudiantil Universitaria en Uruguay Instituto Internacional para la Educación Superior en América Latina y el Caribe. Recuperado de: http://www.cse.edu.uy/sites/www.cse.edu.uy/files/documentos/CSE Resumen Ejecutiv o Boado.pdf

Canales, Andrea, de los Ríos, Danae. (2007) Factores explicativos de la Deserción Universitaria. Revista Calidad de la Educación, (26), 171-201. 
Castaño, Elkin, Gallón, Santiago, Gómez, Karoll y Vásquez Johanna. (2004). Deserción estudiantil universitaria: una aplicación de modelos de duración. Lectura de Economía. (60), 30-65.

Castillo, Mario. (2008). Tasas de deserción en la Universidad Estatal a Distancia de Costa Rica. Revista Electrónica Actualidades Investigativas en Educación, 8 (1), 1-32. Recuperado de http://revista.inie.ucr.ac.cr/buscararticulos/controlador/Article/accion/show/articulo/tasas-de-desercion-en-la-universidadestatal-a-distancia-de-costa-rica.html

Giovagnoli, Paula I. (2002). Determinantes de las deserción y graduación universitaria: Una aplicación utilizando modelos de duración (documentos de trabajo Nro.37). Recuperado de http://www.depeco.econo.unlp.edu.ar/doctrab/doc37.pdf

Hernández, Roberto, Fernández, Carlos y Batista Pilar. (2004). Metodología de la investigación (3a․ ed.) Toluca, México: Mc GrawHill.

Himmel, Erika. (2009). Modelos de análisis de la deserción estudiantil en la Educación superior. Recuperado de http://intranetcolaboradores.inacap.cl/tportal/portales/tp4964b0e1bk102/uploadlmg/File/EducacionSu perior/desercion/1 \%20DesercionE Himmel.pdf

Molina, María Lorena y Ruiz, Ana Isabel. (2001). La gestión del proceso de autoevaluación y la autorregulación para la acreditación de la carrera de Trabajo Social: 1998-2000. Revista de Ciencias Sociales, (92), 123-143.

Programa Estado de la Nación en Desarrollo Humano Sostenible. (2005). Estado de la Educación costarricense (Informe 1). San José, Costa Rica: CONARE.

Programa Estado de la Nación en Desarrollo Humano Sostenible. (2008). Estado de la Educación costarricense (Informe 2). San José, Costa Rica: CONARE.

Regueyra, María Gabriela. (2010a). La Permanencia y graduación de la población estudiantil de Trabajo Social de la Universidad de Costa Rica (Informe Final del proyecto de investigación \#724-B0-142). San José, Costa Rica: Instituto de Investigaciones en Educación, Universidad de Costa Rica.

Regueyra, Gabriela (2010b). La Permanencia y graduación de la población estudiantil de Trabajo Social de la Universidad de Costa Rica (Informe parcial del Proyecto de investigación \#724-B0-142). San José, Costa Rica: Instituto de Investigación en Educación, Universidad de Costa Rica.

Regueyra, María Gabriela y Rojas, Carolina. (2011a). De la deserción a la permanencia: Una propuesta de seguimiento a la población estudiantil universitaria. Ponencia presentada en el "Il Congreso de Investigación Educativa: su incidencia en la realidad" organizado por el Instituto de Investigaciones en Educación (INIE), 1-3 de febrero. San José, Costa Rica: Universidad de Costa Rica. 
Regueyra, María Gabriela y Rojas, Carolina. (2011b). Seguimiento a la permanencia de la población estudiantil de la Escuela de Trabajo Social. Ponencia presentada en las "I Jornadas Interdisciplinarias de Investigación en el Área de Ciencias Sociales", 30 de agosto al 2 de setiembre. San José, Costa Rica: Universidad de Costa Rica.

Rodríguez, Javier y Hernández, Juan M. (2008). La deserción escolar universitaria en México, la experiencia de la Universidad autónoma Metropolitana campus Iztapalapa. Revista Electrónica Actualidades Investigativas en Educación, 8(1), 1-30. Recuperado de http://revista.inie.ucr.ac.cr/uploads/tx magazine/deserc.pdf

Universidad de Costa Rica, Escuela de Trabajo Social. (2006). Plan de Mejoramiento de la Carrera de Bachillerato y Licenciatura en Trabajo Social de la Universidad de Costa Rica 2006-2014. San José, Costa Rica: Escuela de Trabajo Social, Universidad de Costa Rica.

Universidad de Costa Rica, Escuela de Trabajo Social (2009). Acta II-2009 de Asamblea de la Escuela de Trabajo Social realizada el 22 de abril de 2009. San José, Costa Rica: Escuela de Trabajo Social, Universidad de Costa Rica. 